\title{
Different Edge Detection Algorithms Comparison and Analysis on Handwritten Chinese Character Recognition
}

\author{
G. Hemantha Kumar ${ }^{1}$ \\ DoS in Computer Science, \\ University of Mysore, \\ Mysore-570 006, India
}

\author{
Tian Jipeng ${ }^{2}$ \\ DoS in Computer Science, \\ University of Mysore, \\ Mysore-570 006, India
}

\begin{abstract}
The acquired image will has different levels of noise pollution and image distortion in Handwritten Chinese character recognition document image processing. For these situations the accurate and fast edge detection method is an important prerequisite for the recognition results. The widely used edge detection algorithms such as: first derivative-based edge detection method, second derivative edge detection method, canny operator, mathematical morphology edge detection and fuzzy edge detection method. In this paper according to our database of handwritten Chinese characters a lot of experiments to compare the excellent of various algorithms in the field of Chinese character handwriting recognition application. These algorithms are sensitive to image noise and we nowadays discuss new edge detection for the theory of wavelet edge detection method and genetic algorithms. According to our experimental results we explore the new edge detection method to improve the edge detection, image segmentation accuracy and quickness.
\end{abstract}

\section{Keywords}

Edge detection, handwritten Chinese characters, wavelet transform, fuzzy theory, canny operator

\section{INTRODUCTION}

Effective preprocessing method is an important factor to ensure the recognition rate of whole OCR system. Preprocessing has text layout segmentation, line segmentation, and character segmentation. Because of the skew angle while scanning or writing, it makes the characters in input image skew. The skew characters will seriously affect the recognition results. Therefore, character skew detection and transformation should be done before recognition. There are many methods have been developed in this area. [1][3][4][18] Edge detection is an important step in skew correction process.

The edge of the image around the pixel gray is a step change or a change in the roof-shaped collection of pixels. It exists in the target and background, objectives and goals, region to region between the primitive and the primitives. Direction and magnitude of the edge has two features. Along the edge of the trend the pixel value changes gently. Direction perpendicular to the edge of the pixel value changes more violent and may show the step-like or showing slopes. Therefore the edge is divided into two kinds: one is the step of edge, which both sides of the pixel gray value is significantly different; the other is like the edge of the roof, which increased less in the gray value changes from the turning point. For the step of edge the second directional derivative has zero-crossing. For the shape of the roof edge, second derivative in the direction of the edge has the extreme value. How fast and accurate extraction of edge information has been a hot research at home and abroad. However, handwritten Chinese character edge detection is a difficult problem in image processing[2]. Early classical algorithms include edge operator method, surface fitting and template matching method. Now with the development of artificial intelligence and mathematical theory there are many new edge detection methods such as wavelet transform, base and mathematical morphology, fuzzy theory and neural network edge detection methods.

\section{ANALYSIS OF THE CLASSICAL EDGE DETECTION ALGRITHM}

\subsection{The first derivative-based edge detection method}

Current application is more based on differential coefficient edge of the edge extraction algorithm. The gradient operator is one of them. The gradient of the image is $\nabla f(x, y)=\frac{\partial_{f}}{\partial_{x}} i+\frac{\partial_{f}}{\partial_{y}} j$. The $\nabla f(x, y)$ includes gray scale information. The $\mathrm{e}(\mathrm{x}, \mathrm{y})=\sqrt{\mathrm{f}_{\mathrm{x}}^{2}+\mathrm{f}_{\mathrm{y}}^{2}}$ is recorded as the gradient of the edge $\nabla f(x, y)$. The detection operator is $\mathrm{e}(\mathrm{x}, \mathrm{y})$. To make the calculation simply defined $e(x, y)$ as the absolute value of partial derivatives and the sum of $f_{x}$ and $f_{y}$ is $e(x, y)=\left|f_{x}(x, y)\right|+\left|f_{y}(x, y)\right|$.

According to the different size and weight of template there are a lot of gradient operators. The simplest gradient operator is a Roberts operator. It is a $2 * 2$ template using partial differential to detect the edge of steep. But it is more sensitive to noise and always has outliers[5][8]. Then people have developed a Prewitt operator and Sobel operator. Before the two gradient operator get gradient the first do the areas of average or weighted average and then the differential to suppress noise. But it is prone to edge blurring. Then there comes the Kirsch operator uses 8 average gradient operator $(3 * 3)$ image convolution. It gets the maximum as output, which can detect the edge in all directions and reducing the average result of the loosing detail. But at the same time increase the amount of computation. Operator template shows inFigure1. 


\section{$\left[\begin{array}{cc}1 & 0 \\ 0 & -1\end{array}\right]\left[\begin{array}{ll}0 & 1 \\ 1 & 0\end{array}\right] \quad\left[\begin{array}{lll}-1 & 0 & 1 \\ -2 & 0 & 2 \\ 1 & 0 & 1\end{array}\right]\left[\begin{array}{ccc}-1 & -2 & -1 \\ 0 & 0 & 0 \\ 1 & 2 & 1\end{array}\right] \quad\left[\begin{array}{lll}-1 & 0 & 1 \\ -1 & 0 & 1 \\ -1 & 0 & 1\end{array}\right]\left[\begin{array}{lll}1 & 1 & 1 \\ 0 & 0 & 0 \\ 1 & 1 & 1\end{array}\right]$}

Roberts operator Sobel operator Prewitt operator

$$
\begin{gathered}
{\left[\begin{array}{ccc}
5 & 5 & 5 \\
-3 & 0 & -3 \\
-3 & -3 & -3
\end{array}\right]\left[\begin{array}{ccc}
3 & 5 & 5 \\
-3 & 0 & 5 \\
-3 & -3 & -3
\end{array}\right]\left[\begin{array}{ccc}
-3 & -3 & 5 \\
-3 & 0 & 5 \\
3 & 3 & 5
\end{array}\right]\left[\begin{array}{ccc}
-3 & -3 & -3 \\
-3 & 0 & 5 \\
3 & 5 & 5
\end{array}\right]} \\
{\left[\begin{array}{lll}
5 & 5 & 5 \\
3 & 0 & 3 \\
5 & 5 & 5
\end{array}\right]\left[\begin{array}{ccc}
-3 & -3 & -3 \\
5 & 0 & -3 \\
5 & 5 & -3
\end{array}\right]\left[\begin{array}{ccc}
5 & 3 & 3 \\
5 & 0 & -3 \\
5 & -3 & -3
\end{array}\right]\left[\begin{array}{ccc}
3 & 5 & -3 \\
5 & 0 & 3 \\
3 & 3 & 3
\end{array}\right]}
\end{gathered}
$$

Kirsch operator

Fig 1: Frequently used Edge detection operator module

\subsection{The second derivative-based edge detection method}

First derivative is a component of the gradient vector. It not only has size and direction but compare with scalar the data storage is larger. Lap lacian operator is the second derivative operator on two-dimensional function. It has nothing to do with the direction of orientation and not sensitive [4]. So calculation is small. According to the characteristics of the edge the Lap lacian operator can be used as edge detection operator to calculate the value of digital image. Lap lacian can make use of templates (Figure 2). But it is very sensitive to noise, which is equivalent to high pass filter and often there will be some false edges. Therefore, Marr made the first image smoothing with a Gauss function and then use the Lap lacian smooth operator on the images obtained by seeking second derivative zero-crossing point as a candidate edge. This is the LOG operator. LOG operator is the image filtering and differentiation processes. It is the use of rotational symmetry of the LOG of the template and image to do convolution [9]. It determines the filter output zero-crossing position. The template is shown in Figure 2

$$
\begin{aligned}
& {\left[\begin{array}{ccc}
-1 & -1 & -1 \\
-1 & 8 & -1 \\
-1 & -1 & -1
\end{array}\right] \quad \text { Lap lacian Operator }} \\
& {\left[\begin{array}{ccccc}
-2 & -4 & -4 & -4 & -2 \\
-4 & 0 & 8 & 0 & -4 \\
-4 & 8 & 24 & 8 & -4 \\
-4 & 0 & 8 & 0 & -4 \\
-2 & -4 & -4 & -4 & -2
\end{array}\right] \quad \text { LOG Operator }}
\end{aligned}
$$

Fig 2: Lap lacian and LOG operator Module

\subsection{Canny operator}

Canny operator is a filtering, enhancement and optimization of multi-stage detection operator [6]. Before treatment the Canny operator first use Gaussian smoothing filter to smooth the image to remove noise (i.e. with a Gaussian smoothing filter with the image as a convolution.) The edge of the field is enhanced intensity values and highlights the changing point. It is usually done by calculating the gradient magnitude. Canny segmentation algorithm uses first-order partial derivatives of the finite difference to calculate the gradient magnitude and direction. As an edge the first order derivative in the boundary is an upward step or the second derivative zero-crossing. The process of Canny algorithm will go through a process of non- maxima suppression. Last Canny algorithm will be used to connect to the edge of the two thresholds.

\section{NEW EDGE DETECTION ALGORITHMS IN THE APPLICATION OF HANDWRITTEN CHARACTER RECOGNITION}

\subsection{The wavelet transform and wavelet packet approach to edge detection}

In recent years, wavelet analysis [7] is the rapid development of a new field in application mathematic and engineering disciplines. Wavelet transform is the time domain - the local frequency domain transformation, for it can be more effective in extracting information from the signal. In the image project the images often need to analyze complex structures and shapes. It is not only to reflect the contours image edge but not ignore the local details, which requires multi-scale edge detection. The wavelet transform has the natural multi-scale features by shifting, stretching detailed analysis of the signal to achieve the high-frequency breakdown of time and low frequency at the frequency of breakdown [11][12]. Therefore the wavelet transform is very suitable for complex image edge detection.

As an image the most of image energy is concentrated in low and intermediate frequency part. The image edges and noise corresponds to the high frequency part. Based on edge detection surrounded by the wavelet is use of the wavelet function decomposition of the image. In the wavelet transformation only decomposes on the low frequency and not high-frequency sub band of image. Wavelet packet transformation not only analyses the low frequency sub-band but also decomposes the image of the high frequency subband. The larger scale of the wavelet packet is chosen the lower spatial resolution corresponding to wavelet coefficients will get. Therefore the wavelet packet decomposition is a more sophisticated method to meet the different resolution edge extraction. Especially for images with noise it is better for the extracted edge noise reduction.

\subsection{Based on mathematical morphology edge detection method}

The mathematical morphology [10][13] in image processing and pattern recognition is a new discipline with a rigorous mathematical theory and it is now widely used in the image project. The basic idea is to use a certain form of structural elements to measure and extract the morphological structure of the corresponding images to achieve the purpose of image analysis and recognition. The size and shape of structural elements and image structural information have a relationship. It constructs different structural elements and can perform different image analysis. Morphology includes binary morphology, grayscale morphology of color morphology. The basic transformation includes dilation, erosion, opening and closing operations and evolved by the four operators of the open, closed, thinning, thickness and so on. Thus it completes the complex morphological transformation. With the binary method is currently used more and more mature the grayscale and color morphology in edge detection applications are increasingly a cause for concern and gradually.

\subsection{Edge Detection Based on Fuzzy Methods}


The fuzzy theory [14][15] was founded in 1965 by the U.S. Department of Electrical Engineering. University of California Professor Zadeh fuzzy brings forward the theory based on the theory proposed. The characteristics of fuzzy theory is not check the simple things whether positive or negative but use degree of membership to reflect the scope of a thing belonging to a certain extent. In the imaging system the visual reflection of the ambiguity caused by the image itself add the edge of the ambiguity of the definition of distinction then the people naturally deal with images fuzzy theory. The more representative for foreign scholars Pal and King proposed fuzzy edge detection algorithm. The central idea is to increase the use of fuzzy Enhancement contrast between different regions, which can extract fuzzy edges. The advantage of Edge detection based on fuzzy theory method is its mathematical basis. The disadvantage is the calculation involves matrix inverse transform and more complex operations such as addition. It not only increases contrast but also increases the noise

\section{COMPARISON OF TEST RESULTS}

Several edge detection operators for edge detection image database sample results are shown in Figure 3. Roberts edge operator extracts coarse edge and location is not accurate. Some thin edge of the drop may be neglected. Sobel operator and Prewitt operator can get more precise positioning on the edge. LOG operator and Canny edge detection operator on the result was better than the first three operators. Especially it has more complete extraction of the edges and more accurate position can be detected. The edge of the image is smaller. The classical edge detection operator of is simpler, mature, fast and the disadvantage is more sensitive to noise. However, an improved one is considerable potential for application. Several new edge detection algorithms in the field of Handwritten Chinese character recognition applications are increasingly widespread[16][17][18].

Although the field of classical edge detection has the edge detection operator and the other detections are based on wavelet, fractal theory method and morphology. But they are not the method has an absolute advantage and some edge detections have high precision but reduction noise is poor. Something solves the problem of poor anti-noise performance but detection accuracy was not well. Although there's a better solution to a certain extent of these two agreements but computation is complex and computing time is long. Whatever the edge detection algorithm in solving certain problems is at the same time there are different types of defects. Thus searching a simple algorithm, a better solve the edge detection accuracy, a coordination of anti-noise performance of edge detection algorithm image processing and analysis has been one of the purposes of image processing. There are still a lot of works to do.

\section{CONCLUSION}

The edge detection for handwritten Chinese character recognition is a very importance of the process. In this paper we experimented and compared various algorithms. Canny operator and mathematical based on morphology has better detection rate and interference. Although each algorithm can not well balanced performance and noise detection accuracy but the handwritten Chinese characters based on contributions collected from the noise characteristics and take the appropriate edge detection algorithm will be able to achieve the desired effect. In the future we will explore how to take the initiative to explore the noise pollution, which automatically selects the edge detection algorithm and then improve the manuscript handwritten Chinese character recognition.

\section{REFERENCE}

[1] Wang Zhengyao, Digital Image Edge Detection, Master Thesis, Xian Transportation University, 2003

[2] Lei Lizhen, Digital Image Edge Detection Analysis, Journal of Image Processing, 2006. 3, 40-42

[3] Zheng Nanning, Computer Vision and Pattern Recognition, Beijing, National Security Publish, 1998

[4] Li Xiaohong, Research on LOG filter of Image edge detection, Computer Application and Software, 2005,22(5), 107-108

[5] Chen Hongxi, Canny operator edge detection, Wuhan Institute University Journal, 2006,25(1),86-90

[6] Ma Yan, Comparison of different edge detection operator, Engineer automation, 2004, (1) 54-56

[7] Cheng Zhengxing, Wavelet using in image processing, Engineer Math Journal, 2001,(1),57-86

[8] Wang Jianzhong, Improving on wavelet using in image edge extraction, Lanzhou University Journal, 2004,26(1):76-79

[9] Zhao Dengfeng, Wavelet using in Image Processing, Tongji University journal, 2001, 29(9): 1054-1057

[10] Cui Qi, Image Processing and Analysis-mathematical morphology application, Beijing: Science Publish, 2000

[11] Feng Junping, Edge detection based on Mathematical morphology, Computing technology, 2004, 34(3):53-56

[12] Wang Shuwen, Mathematical morphology using in image processing, Computer engineer and application, 2004, 3:89-92

[13] Yang Pingxian, Improved edge detection algorithm, Optical Engineering, 2005, 32(11):72-75

[14] Zhang Shihua, Based on Fuzzy extraction increasing edge detection, Hunan Engineering College Journal, 2006, 65-68

[15] Shi Chengxiang, Extended multi scale fuzzy edge detection, Computer Engineering and application, 2006, $65-67$

[16] Bai Jianming, Fractal Theory using in X-ray picture edge detection, Heilongjiang Medical Science, 2006, 29(1): 78-79

[17] Cui Xudong, Using marking relaxation method on flash image edge detection, Optical Engineer, 2001, 28(4): $42-$ 45

[18] Xiao feng, Base on BP neural network research on image edge detection algorithm, Xian Technology University Journal,2005,25(3):372-375. 
Original Image

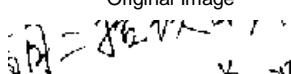

国务院说理

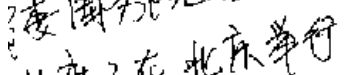

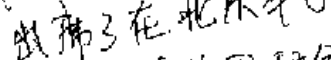

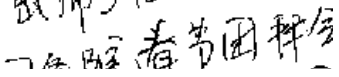

Roberts

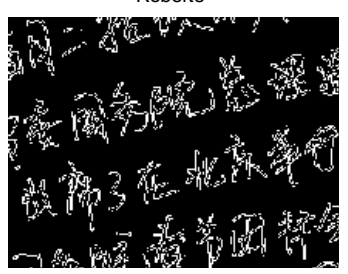

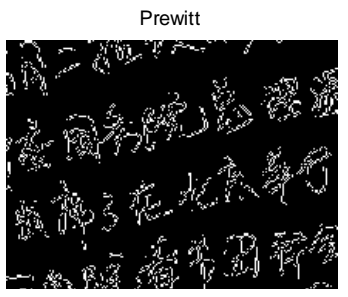
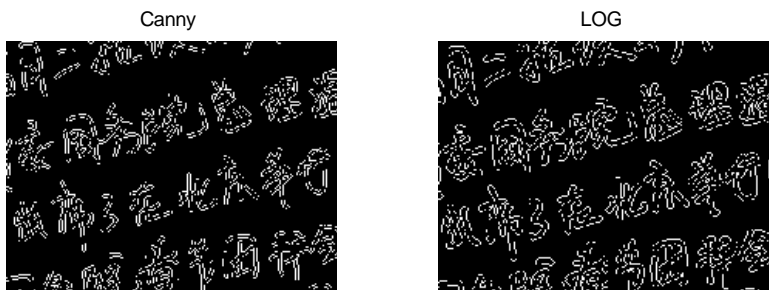

Math
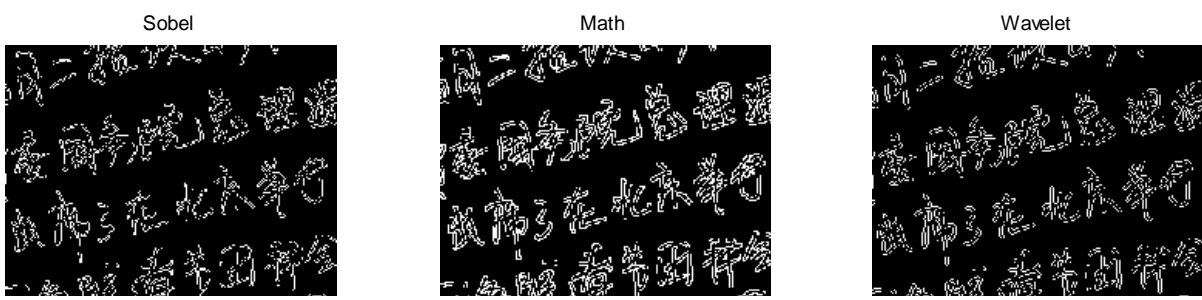

Fig 3: Experiment results on different operators 